MATEC Web of Conferences 22,05011 (2015)

DOI: $10.1051 /$ matecconf/ 20152205011

(C) Owned by the authors, published by EDP Sciences, 2015

\title{
Economic Dispatch Considering Correlations of Solar and Load
}

\author{
Xiaohong Ran*, Shihong Miao \& Hao Xu \\ State Key Laboratory of Advanced Electromagnetic Engineering and Technology, Huazhong University of Sci- \\ ence and Technology, Wuhan, Hubei, China
}

\begin{abstract}
An economic dispatch considering correlation between solar and load is proposed. The probabilistic density functions of solar and load are introduced, and then solar-load bivariate normal distribution is established based on multivariate statistical analysis technique. The loss load risk model of typical scenario is constructed and the economic dispatch model is established. $\alpha$-superquantile is used to describe the risk constraint, Monte Carlo simulation and analytic method were combined to solve the optimization model. Results show that proposed method can describe the impact of correlation of solar and load on economic dispatch at different confidence levels.
\end{abstract}

Keywords: correlation; risk model; economic dispatch; unit commitment

\section{INTRODUCTION}

Photovoltaic generation (PV) has gradually become one of the most potential power generation technologies of renewable energy. Currently, the scale of PV is still expanding, it predicts that the global PV capacity will be increased to $180 \mathrm{GW}$ in 2015 , while PV will account for $9 \%$ of global power demand in 2030 [1]. However, random factors of PV such as the power output and the load prediction error can affect the system stable operation [2]. Therefore, it is very important to study the influence of correlation between solar and load on economic dispatching of power system.

Volatility of PV limits its grid-connected capacity, Yang [3] puts forward a method of PV power's penetration limit based on peak load balance constraint, but it doesn't consider the influence of correlation of PV power. Wang [4] considers the complementarity of wind power, PV and energy storage, and evaluates reliabilities on different coordinated operation strategies, but it also doesn't involve the correlation between wind power and PV. Sun [5] establishes a joint probabilistic model based on correlation of wind speed and load, and puts forward a reliability evaluation method of distribution networks. Until now, there is little study about the influence of correlation between the solar power and the net load on economic dispatching.

Recent years, chance-constrained programming is widely used in optimization problems of power system. $\mathrm{Wu}[6]$ considers uncertain factors such as the electricity price, the reservoir inflow and the unit operation condition, researches influence on short-term optimization dispatching of cascade hydropower station. Lei [7] focuses on the randomness of wind power and load, installed the capacity of wind power and output of conventional units which are regarded as optimization variables, studies the wind power penetration limit. But the computation burden of optimization models is complex in [6]-[7]. In [8], a complex non-linear model is changed into a linear programming problem using $\alpha$-superquantile method. On one hand, $\alpha$-superquantile method can qualify the potential losses exceeding the assumed confidence level; On the other hand, it is easy to solve the linear programming problem. But method used in [8] focuses on single type of random factor; it's difficult to solve combined effects of several random factors on risk optimization problem.

In this paper, the joint probability distribution functions of solar and load are introduced, the load shed risk model and the economic dispatch model based on different typical scenarios are established. The correctness and efficiency of proposed method are verified by programming in Matlab.

\section{JOINT PROBABILITY DISTRIBUTION MOD- EL OF SOLAR AND LOAD}

The forecasting error of solar is treated as a random variable that follows normal distribution, $\bar{E}$ is a forecasting value of solar, the probability distribution function (PDF) of actual solar is shown as follows:

$$
f_{E}(E)=\frac{1}{\sqrt{2 \pi} \sigma_{E}} \exp \left(-(E-\bar{E})^{2} /\left(2 \sigma_{E}^{2}\right)\right)
$$

Where, the variance of forecasting solar is $\sigma_{E}^{2}=\bar{E} \times \omega \%$.

The solar power output mainly depends on solar radiation, and the solar-to-power function is shown as follows:

$E_{a v}(t)=E \eta^{P V} S^{P V}$

*Corresponding author: ranxhcsust@126.com 
Where, $\eta^{P V}, S^{P V}$ and $E_{\alpha v}$ are respectively the efficiency, solar area $\left(\mathrm{m}^{2}\right)$ and solar power output. In addition, the load has forecasting error, and corresponding PDF is given in [9].

In this paper, forecasting values of solar radiation and load respectively follow the Gaussian (GD) distribution, the linear combination $l_{1} x_{1}+l_{2} x_{2}$ follows one-dimension, so the random variable $\left(x_{1}, x_{2}\right)^{T}$ follows two-dimension GD [5].

$$
\begin{aligned}
& f\left(x_{1}, x_{2}\right)=\frac{1}{2 \pi \sigma_{1} \sigma_{2} \sqrt{1-\rho^{2}}} \times \exp \left\{-\frac{1}{2\left(1-\rho^{2}\right)}\left[\frac{\left(x_{1}-\mu_{1}\right)^{2}}{\sigma_{1}^{2}}\right.\right. \\
& \left.\left.-2 \rho \frac{\left(x_{1}-\mu_{1}\right)\left(x_{2}-\mu_{2}\right)}{\sigma_{1} \sigma_{2}}+\frac{\left(x_{2}-\mu_{2}\right)^{2}}{\sigma_{2}^{2}}\right]\right\},-\infty<x_{1}, x_{2}<+\infty
\end{aligned}
$$

Where, $\mu_{1}, \mu_{2}$ and $\sigma_{1}, \sigma_{2}$ are means and standard deviations of $x_{1}, x_{2}, \rho$ is a correlation of $x_{1}, x_{2}$.

According to the solar-to-power conversion, the inverse function of solar power and load is shown as follows:

$\left\{\begin{array}{l}E=E_{a v} /\left(\eta^{P V} S^{P V}\right) \\ \Delta P_{D}=\Delta P_{D}\end{array}\right.$

The Jacobi matrix of (4) is given by:

$J=\left|\begin{array}{ll}\frac{\partial E}{\partial E_{a v}} & \frac{\partial E}{\partial \Delta P_{D}} \\ \frac{\partial \Delta P_{D}}{\partial E_{a v}} & \frac{\partial \Delta P_{D}}{\partial \Delta P_{D}}\end{array}\right|$

Therefore, the joint PDF of random variables $U=\left(E_{a v}, \Delta P_{D}\right)$ is shown as follows:

$g\left(E_{a v}, \Delta P_{D}\right)=|J| f\left(E_{a v} /\left(\eta^{P V} S^{P V}\right), \Delta P_{D}\right)$

\section{SUPERQUANTILE QUALIFICATION AND LOAD SHED RISK MODEL}

To quantify different types of uncertainties in engineering problem conveniently, R. T. Rockafellar firstly presented a superquantile method in 2010 , which was a proposed independent application named $\alpha$-superquantile[10]. First, it can qualify potential losses at an assumed confidence level; second, it enlarges the range of usage.

Let $g(\boldsymbol{x}, w, v)$ be a loss function depending on the decision vector $\boldsymbol{x} \in \boldsymbol{X} \subset \boldsymbol{R}^{n}$ and the random vector $w, v$ whose PDF is $\rho(w, v)$. The probability doesn't exceed a threshold $a$ which is shown as follows:

$\psi(\boldsymbol{x}, \alpha)=\iint_{g(\boldsymbol{x}, w, v) \leq \alpha} \rho(w, v) d w d v$

For confidence level $\beta$, the $\alpha$-quantile is defined as follows:

$q_{\alpha}(\boldsymbol{x})=\min \{\alpha \in \boldsymbol{R}: \psi(\boldsymbol{x}, \alpha) \geq \beta\}$
Because $\alpha$-quantile can't deal with extent of losses that might be suffered beyond threshold, $\alpha$-superquantile could qualify the potential risk losses, and expression is shown as follows:

$\bar{q}_{\alpha}(\boldsymbol{x})=\frac{1}{1-\beta} \int_{g(\boldsymbol{x}, w, v) \geq q_{\alpha}(\boldsymbol{x})} g(\boldsymbol{x}, w, v) \rho(w, v) d w d v$

It can be seen from (9), $q_{\alpha}(\boldsymbol{x})$ is included in formula $q_{\alpha}(\boldsymbol{x})$ because $q_{\alpha}(\boldsymbol{x})$ is very difficult to be solved. However, expectation can be estimated by its samples as follows:

$\bar{\eta}_{\beta}(\boldsymbol{x}, \alpha)=\alpha+\frac{1}{n m(1-\beta)} \times$

$\sum_{j=1}^{n} \sum_{k=1}^{m} \max \left\{0, g\left(\boldsymbol{x}, w^{j}, v^{k}\right)-\alpha\right\}$

Where, $m$ and $n$ are the number of discrete points for random variable $w, v$.

As for the load shed risk, two typical modes would be studied: One is without solar and another is with solar. First, the load uncertainty is only considered, and PDF is described as follows:

$$
F_{Z_{P}}(z)=P\{Z \leq z\}=\int_{-\Delta P_{D} \leq z} f_{\Delta P_{D}}\left(\Delta P_{D}\right) d \Delta P_{D}
$$

In addition, when uncertainties of solar power and load forecasting error are considered, the PDF of random variables is written as follows:

$$
F_{Z_{E}}(z)=\iint_{E_{a v}-\Delta P_{D} \leq z} g\left(E_{a v}, \Delta P_{D}\right) d E_{a v} d \Delta P_{D}
$$

The risk of load shed referring to the spinning reserve of power system is less than amount of power fluctuation of solar power and load due to forecasting error

Typical scenario 1: without wind and solar

According to (11), the load forecasting error is only considered, and the load shed risk model is written as follows:

$$
P_{\text {loadshed }}=h_{\text {risk }, u}\left(D_{t}^{u}, \Delta P_{D}\right)=P\left(D_{t}^{u} \leq \Delta P_{D}\right)
$$

Where, $D_{t}^{u}$ is the up spinning reserve of load at period $t$.

Typical scenario 2: wind and solar are complementary

According to (12), the forecasting errors both in solar power and load are considered, and the load shed risk model is given by:

$$
\begin{aligned}
& P_{\text {loadshed }}=h_{r i s k, u}\left(D_{t}^{u}, E_{a c t}, \Delta P_{D}\right) \\
& =P\left(D_{t}^{u} \leq \Delta P_{D}+\left(E_{a c t}-E_{a v}\right)\right)
\end{aligned}
$$

Where, $E_{\text {act }}$ is the actual solar power output.

The larger or smaller load shed risk don't represent higher or lower load loss, thus the load loss is defined as multiplying using the load shed risk and the power fluctuation amount: 


$$
h_{\text {risk,u }}\left(D_{t}^{u}, E_{\text {act }}, \Delta P_{D}\right) \leq \alpha_{1}
$$

Where, $h_{\text {risk,u }}=P_{\text {loadshed }} \times \Delta P_{D}, \quad \alpha_{1}$ is a given risk limit.

\section{ECONOMIC MODEL CONSIDERING SEVER- AL UNCERTAINTIES}

\subsection{Objective functions}

The generation cost and the starting-up cost of units are considered in the dispatch model, thus the objective function is stated as follows:

$$
F=\min \left[\sum_{t=1}^{T} \sum_{i=1}^{n} u_{i t} f\left(P_{i t}\right)+\sum_{k=1}^{n} S_{k t}\left(1-u_{i t-1}\right) u_{i t}\right]
$$

Where, $f\left(P_{i t}\right)$ is the generation cost of each unit, $n$ is the number of units, $T$ is the dispatch periods, $u_{i t}=1$ and $u_{i t}=0$ refer to operation and stopping state. In addition, the generation cost of each unit is shown as follows:

$$
f\left(P_{i t}\right)=a_{i} P_{i t}^{2}+b_{i} P_{i t}+c_{i}
$$

Where, $a_{i}, b_{i}, c_{i}$ are the cost coefficient. Additionally, unit commitment is considered, starting-up cost function is shown in [11].

\subsection{System constraints}

1) System constraints are shown as follows:

$$
\left\{\begin{array}{l}
\sum_{i=1}^{n} u_{i t} P_{i t}+E_{a c t}=P_{D t} \\
P_{i t}^{\min } \leq P_{i t} \leq P_{i t}^{\max }, i=1,2, \cdots n \\
-\xi_{d} \leq P_{i t}-P_{i t-1} \leq \xi_{u}, i=1,2, \ldots n \\
T_{i}^{o n}>U T_{i}, T_{i}^{\text {off }}>D T_{i}
\end{array}\right.
$$

Where, $P_{D t}$ is the load demand of period $t, P_{i t}^{\max }$ and $P_{i t}^{\min }$ are maximum/minimum power limits of unit $i$, $\xi_{u}$ and $\xi_{d}$ are maximum/minimum ramp rate limits, $U T_{i}$ and $D T_{i}$ are minimum up/down time of unit $i$.

2) Reserve constraints are shown as follows:

$$
\left\{\begin{array}{l}
D_{t}^{u}=\sum_{i=1}^{n} U S_{i}(t) \geq \sum_{i=1}^{n} U S R_{i}(t)+S R_{1}\left(E_{a v}\right) \\
D_{t}^{d}=\sum_{i=1}^{n} U D_{i}(t) \geq S R_{2}\left(E_{a v}\right)
\end{array}\right.
$$

$$
\begin{aligned}
& \text { Where } \\
& U S_{i}(t)=\min \left\{U S_{i t}^{\max }, P_{i t}^{\max }-P_{i}(t)\right\}, \\
& U S R_{i}(t)=P_{D t} \times L \%, \\
& U S_{i t}^{\max }=d \% \times P_{i t}^{\max }, \\
& U D_{i}^{\max }=d \% \times P_{i t}^{\max }, \\
& U D_{i}(t)=\min \left\{U D_{i}^{\max }, P_{i}(t)-P_{i t}^{\min }\right\},
\end{aligned}
$$

$S R_{1}\left(E_{0}\right)=S R_{2}\left(E_{0}\right)=E \times r \%$

3) Load shed risk constraints are shown as follows:

$\left\{\begin{array}{c}\eta_{\beta_{1}}\left(P_{i t}, E_{a c t}, \Delta P_{D}, \alpha_{1}\right) \approx \alpha_{2}+\frac{1}{n m(1-\beta)} \sum_{j=1}^{n} \sum_{k=1}^{m} z_{j k} \leq \alpha_{1}(20) \\ z_{j k r}(t) \geq 0, z_{j k r}(t) \geq h_{\text {risk,u }}-\alpha_{2}\end{array}\right.$

Where, $\alpha_{2}$ is the $\alpha$-quantile of load loss.

\section{CASE STUDY}

In this case, ten units of system are considered to be studied to validate effectiveness of proposed method [11]. The parameters of efficiency of photovoltaic $\eta^{P V}$ $=12 \%, S^{P V}=3000 \mathrm{~m}^{2}$, and the dispatch period is three hours, the power base is 100 MVA. Predictions of solar radiation are shown in Table 1 where $\omega \%=8 \%$, $d \%=12 \%, L \%=9 \%, r \%=10 \%$.

Table 1. Parameters of solar radiation.

\begin{tabular}{l|l|l|l|l}
\hline Typical mode & Parameters & Period1 & Period2 & Period3 \\
\hline Without solar & $E\left(\mathrm{~W} / \mathrm{m}^{2}\right)$ & 0.2 & 0.4 & 0.6 \\
\hline \hline With solar & $E\left(\mathrm{~W} / \mathrm{m}^{2}\right)$ & 463 & 470 & 465 \\
\hline
\end{tabular}

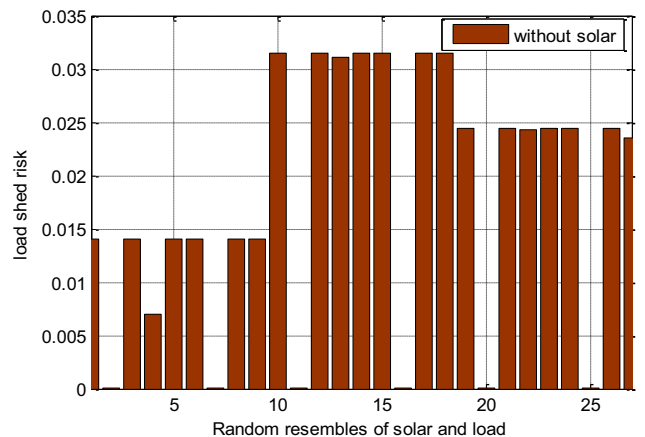

Figure 1. Load shed risk of without solar mode

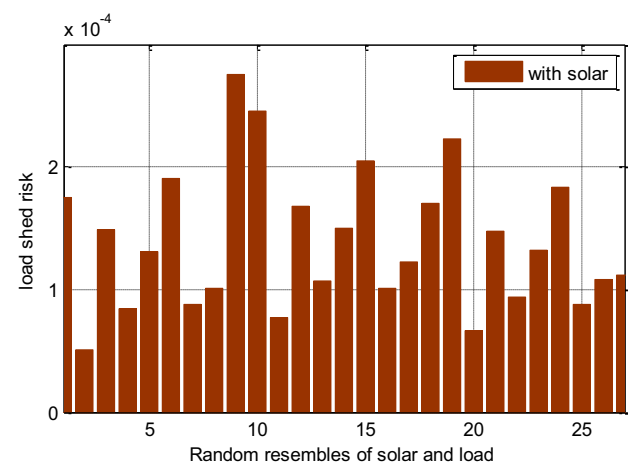

Figure 2. Load shed risk of with solar mode.

The results of load shed risk in different typical op- 
MATEC Web of Conferences

\begin{tabular}{|c|c|c|c|c|c|c|c|c|}
\hline \multirow{2}{*}{ Periods } & \multirow[b]{2}{*}{$\alpha_{i}$} & \multicolumn{7}{|c|}{ Power output/pu } \\
\hline & & $\mathrm{G} 1$ & G2 & G3 & G4 & G5 & G6 & G7 \\
\hline \multirow{3}{*}{1} & 0.000090 & 1.5000 & 0.0000 & 1.0000 & 0.7000 & 1.2000 & 3.2729 & 4.7500 \\
\hline & 0.000126 & 1.5000 & 0.0000 & 1.0000 & 0.7000 & 1.2000 & 3.1422 & 4.7500 \\
\hline & 0.000162 & 1.5000 & 0.0000 & 1.0000 & 0.7000 & 1.2000 & 3.0115 & 4.7500 \\
\hline \multirow{3}{*}{2} & 0.000090 & 1.2000 & 0.7000 & 1.0000 & 0.9000 & 2.9729 & 4.4500 & 2.8589 \\
\hline & 0.000126 & 1.2000 & 0.7000 & 1.0000 & 0.9000 & 2.8422 & 4.4500 & 2.8432 \\
\hline & 0.000162 & 1.2000 & 0.7000 & 1.0000 & 0.9000 & 2.7115 & 4.4500 & 2.8274 \\
\hline \multirow{3}{*}{3} & 0.000090 & 1.2113 & 0.4000 & 1.0000 & 0.6000 & 2.6729 & 4.1500 & 3.1589 \\
\hline & 0.000126 & 1.1959 & 0.4000 & 1.0000 & 0.6000 & 2.5422 & 4.1500 & 3.1432 \\
\hline & 0.000162 & 1.1806 & 0.4000 & 1.0000 & 0.6000 & 2.4115 & 4.1500 & 3.1274 \\
\hline
\end{tabular}

erational scenarios are shown in Figure 1 and Figure 2. It can be seen from Figure 1 that the uncertainty mainly consists of load forecasting error, and the maximum load shed risk is about 0.032 for it is without solar typical operational mode. From Figure 2, uncertainty mainly consists of the fluctuation of solar power and the load forecasting error. The maximum load shed risk is about 0.0003 for it is with solar operational mode. Compared Figure 1 with Figure 2, it can be seen that the load shed risk without solar mode is larger than the ones with solar mode. Thus, with solar mode, it is good for reduction towards the load shed risk.

The sequential quadratic programming is used to solve economic dispatch model. Table 2 shows dispatch results with solar at the confidence level of 0.95 . From Table 2, in addition to the fixed power output, the power output of units would be decreased with the increase of risk limit.

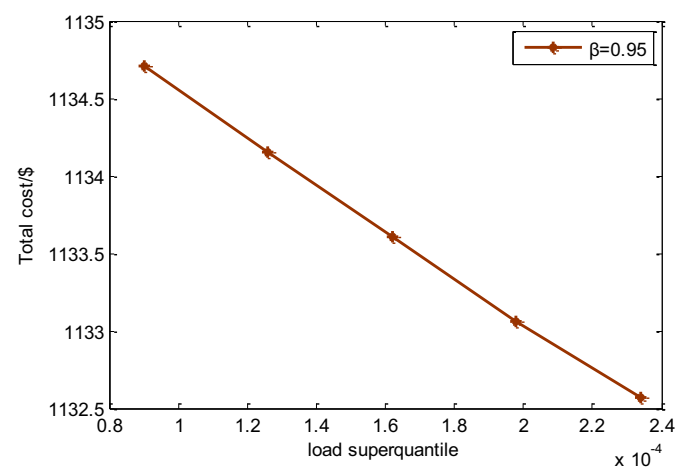

Figure 3. Variation of cost with confidence level

Figure 3 shows the tendency of total cost with the load superquantile. From Figure 3, objective values would be decreased with increase of $\alpha$-superquantile of load loss, because the reliability of power system loads is decreased, the total cost would be decreased accordingly.

\section{CONCLUSIONS}

1) This paper presents a new method to quantify combinations of solar power and net load, while the load shed risk and the economic dispatch model are estab- lished in different typical scenarios. The dispatch model could provide useful message for making a suitable dispatch strategy.

2) The complex nonlinear constraints of load loss are changed into linear constraints by $\alpha$-super quantile method which is very useful to solve the economic dispatch model quickly and accurately.

\section{ACKNOWLEDGEMENT}

This paper is sponsored by National Natural Science Foundation of China (GN: 51377068)

\section{REFERENCES}

[1] Liang Shuang, Hu Xuehao, et al. 2011. Current status and development trend on capacity credit of photovoltaic generation. Automation Electric Power Systems, 35(19): 101-107.

[2] Ding Ming, Wang Weisheng, Wang Xiuli, et al. 2014. A review on the effect of large-scale PV generation on power systems. Proceedings of the CSEE. 34(1): 1-14.

[3] Yang Nan, Liu Dichen, Sun Wentao, et al. 2013. Research about photovoltaic power's penetration limit based on the peak load balance constraint. Power System Protection and Control. 41(4): 1-6.

[4] Wang Haiying, Bai Xiaomin, et al. 2012. Reliability assessment considering the coordination of wind power, solar energy and energy storage. Proceedings of the CSEE, 32(13): 13-20.

[5] Sun Ruodi, Xie Kaigui. 2012. Reliability evaluation of distribution networks using Monte Carlo method considering correlations between wind speed and load. Power System Protection and Control. 40(18): 12-18.

[6] Wu Jiekang, et al. 2008. A new strategy for short-term scheduling optimization of cascade hydro plants based on chance-constrained programming. Proceedings of the CSEE. 28(13): 41-46.

[7] Lei Yazhou, Wang Weisheng, et al. 2002. Wind power penetration limit calculation based on chance considering programming. Proceedings of the CSEE. 22(5): 32-35.

[8] Liu Yangsheng, Zhou Renjun, Li Xinglang, et al. 2013 Inside-plant optimal operation of carbon capture unit under carbon emission right trade. Power System Technology, 37(2): 295-300. 
ICETA 2015

[9] Su Peng, Liu Tianqi, Li Xingyuan. 2010. Determination of optimal spinning reserve of power grid containing wind. Power System Technology. 34(12): 158-162.

[10]R.T. Rockafellara \& J.O. Royset. 2010. On buffered failure probability in design and optimization of structures. Reliability Engineering and System Safety. 95(2010): 499-510.

[11]Li Canbing, Lü Su, Cao Yijia, et al. 2012. A new method for day-ahead unit commitment based on energy-saving generation dispatching. Proceedings of the CSEE. 32(16): 70-76. 